\title{
JAMS Uma imagem de marca
}

Pediram-me para escrever um breve apontamento sobre o Professor José Artur Martinho Simões (JAMS) e como ele influenciou a minha vida cientifica. Sem querer ser demasiado intimista, aqui fica o relato, na primeira pessoa, do que sinto como essa influência.

Em Março de 1984, comecei a trabalhar na linha II do Centro de Química Estrutural do Instituto Nacional de Investigação Científica (Complexo I do IST) com o Doutor Manuel Eduardo Minas da Piedade, ao tempo aluno de doutoramento do Prof. Martinho Simões. Na altura pretendia-se sintetizar complexos organometálicos de Mo e W com moléculas como $\mathrm{H}_{2}$ e etileno coordenadas de modo $\eta^{2}$. Foi com ele que efectuei a minha primeira "Big Prep" (síntese do hidreto de metal com os respectivos ligandos ciclopentadienilo) e me iniciei nas técnicas de Schlenk e nos compostos sensiveis ao ar que desde então me têm seguido por todo o lado. Foi também nessa altura que conheci o Professor Martinho Simões que, curiosamente, nunca foi meu professor durante a licenciatura no Instituto Superior Técnico.

Recordo desde essa altura a facilidade de contacto e o entusiasmo contagiante que põe nas coisas que faz, e o facto de ter disponibilizado um computador pessoal com impressora (raros nesses anos de 1985/1986) para que eu, o Hermínio Diogo e o Adelino Galvão pudessemos, fora do horário laboral, escrever o nosso Projecto de Indústrias Químicas (Projecto Final de Curso).
Ainda antes de terminar a licenciatura o Prof. Martinho Simões apresentou, a mim e aos meus colegas que com ele trabalhávamos na linha II, várias propostas de futuro pós-licenciatura. Achei algumas delas interessantes e, após alguma concertação, a minha escolha recaiu num projecto conjunto entre o Prof. Martinho Simões e o Doutor Pires de Matos que trabalhava no, na altura, ICEN em Sacavém. Pretendia-se estudar a termoquímica de complexos organometálicos de urânio e tório. Nessa altura o grupo liderado pelo Doutor Pires de Matos tinha já iniciado a síntese e estudo da reactividade de alguns desses compostos em que os tradicionais ligandos estabilizadores ciclopentadienilos haviam sido substituídos por uma outra família de ligandos, os polipirazolilboratos [1]. E foi assim que eu fui para o ICEN (actual Instituto Tecnológico e Nuclear) onde ainda hoje continuo a trabaIhar.

\section{A Termoquímica em Sacavém}

Chegado a Sacavém, os primeiros tempos foram gastos a aprender a usar e manter as caixas de luvas e a tentar sintetizar compostos de urânio do tipo $\mathrm{U}\left[\mathrm{HB}\left(3,5-\mathrm{Me}_{2} \mathrm{pz}\right)_{3} \mathrm{Cl}_{2} \mathrm{X}\right.$ (X=Cl, O-t-Bu, $\mathrm{NEt}_{2}, \ldots$ ) (figura 1). Recordo em especial a ajuda imensa da Doutora Noémia Marques no referente às sinteses e o bom ambiente laboratorial fomentado pela Adelaide Carvalho que tornaram esses tempos, cheios de insucessos laboratoriais, fáceis de suportar (segundo o meu caderno de laboratório a síntese do $\mathrm{U}\left[\mathrm{HB}\left(3,5-\mathrm{Me}_{2} \mathrm{pz}\right)_{3}\right] \mathrm{Cl} 2\left(\mathrm{NEt}_{2}\right)$ foi tentada 16 vezes ... a última delas com sucesso).

Estes compostos são mais sensiveis ao oxigénio e à humidade que os compostos organometálicos de metais de transição com ciclopentadienilos, pelo que se tornou necessário desenvolver uma metodologia diferente para as determinações calorimétricas com os mesmos. Em primeiro lugar algumas sínteses conduziam a quantidades muito pequenas de composto (menos de $250 \mathrm{mg}$ ) o que tornava a sua disponibilidade exígua. Por outro lado seria necessário construir células calorimétricas estanques para calorimetria de solução-reacção e definir protocolos de funcionamento que permitissem manusear esses compostos até ao momento de se efectuar as medidas. Foi necessário estudar as reacções adequadas para os estudos calorimétricos e as condições em que teriam que ser feitas. Levou algum tempo; as dificuldades foram sendo vencidas, as células calorimétricas e restante aparelhagem foram sendo instaladas (figura 2) e em 1990 foram comunicados em "poster" em Gandia (Espanha) e em Como (Itália) os primeiros resultados de ental pias de dissociação U-C e U-N por nós obtidas [2].

Durante estes anos o apoio do Prof. Martinho Simões foi sempre tão constante como discreto, deixando-me encontrar a minha velocidade de cruzeiro e não impedindo, incentivando mesmo, que alguns resultados que começaram por ser laterais pudessem florescer

Departamento de Química, Instituto Tecnológico e Nuclear, 2686-953 Sacavém, Portugal. E-mail: jpleal@itn.pt 

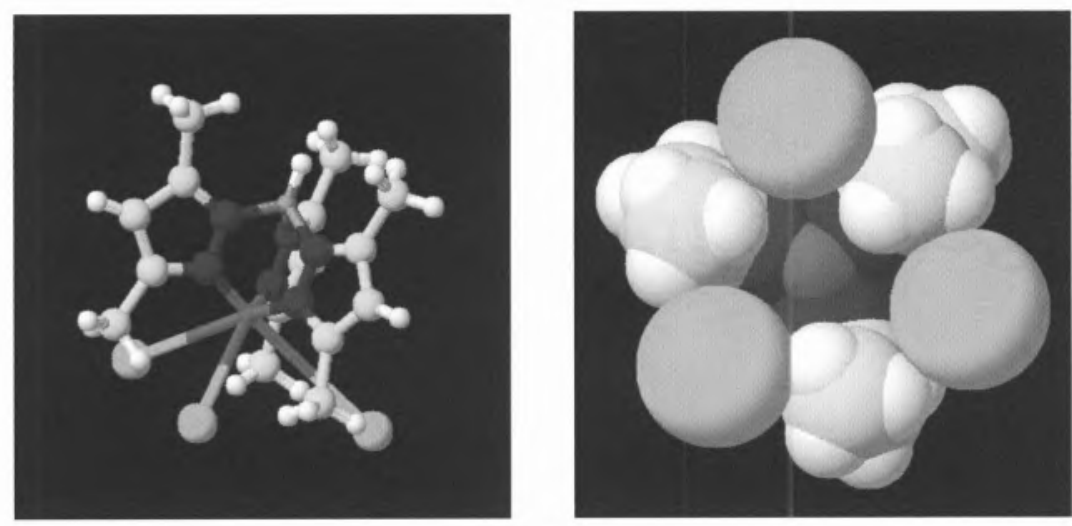

como uma outra familia de compostos a cuja energética tenho desde então dedicado especial atenção: os sais de metais alcalinos e alcalino-terrosos [3]. Nem mesmo a sua ausência de um ano em 1988/89 no Canadá impediram que esse apoio continuasse. Guardo dessa altura algumas cartas que trocámos, partilhando as vitórias e desaires da investigação (parece incrível, mas nessa altura não havia e-mail como hoje acontece). E foi assim que concluí em 1993 o meu doutoramento em energética de compostos organometálicos de urânio e de alcóxidos de metais alcalinos.

Desde essa altura, a um ritmo mais ou menos acelerado, o estudo da energética de compostos de coordenação (no sentido mais vasto do termo) de actinídeos e lantanídeos tem continuado no grupo de Química Inorgânica e Organometálica [4] existindo neste momento um programa de doutoramento em curso sobre a reactividade e energética de compostos de urânio com polipirazolilboratos [5].

Em 1987 concorremos em parceria tripla, ITN (Grupo de Química Inorgânica e Organometálica), IST (grupo de Termoquímica da linha II) e FCUL (Centro de Espectrometria de Massa) a um projecto Ciência para a aquisição de um espectrómetro de massa de ressonância ciclotrónica de iões com transformada de Fourier. Também aqui, o Prof. Martinho Simões esteve activamente envolvido sendo um dos seus proponentes mais entusiastas. Tal aparelho permite, para além da componente analítica de um espectrómetro de massa, estudar a reactividade de iōes com moléculas neutras e equilibrios envolvendo iões

\section{A química na fase gasosa}

figura 1 - Representação gráfica do composto $\mathrm{U}\left[\mathrm{HB}\left(3,5-\mathrm{Me}_{2} \mathrm{pz}\right)_{3}\right]_{\mathrm{Cl}}$. a) vista do composto evidenciando os modos de coordenação; b) representando os átomos com os respectivos raios de Van der Walls uma vista axial mostra o átomo de urânio (a vermelho) com uma esfera de coordenaçâo suficientemente ocupada para ser estável, mas ainda com alguma reactividade. devido às suas capacidades de isolar os iões espacialmente e durante tempos relativamente longos. Os deuses foram favoráveis e o nosso projecto foi financiado, tendo o aparelho sido instalado no Departamento de Química do ICEN (actual ITN) onde continua em operação (figura 3).

No "log-book do aparelho a primeira entrada que se regista é de 17 de Novembro de 1991 e relata que conseguimos ver os iões $\mathrm{Cu}^{+}, \mathrm{U}^{+}, \mathrm{UO}^{+}$e $\mathrm{UO}_{2}^{+}$. Se não me engano, foi o primeiro equipamento do Programa Ciência a estar 100\% operacional. Desde essa data até ao presente a produção do aparelho tem sido intensa, em especial devido à dinâmica introduzida pelos meus colegas Doutores António Pires de Matos e Joaquim Marçalo e uma pleiade de estudantes e utilizadores que por lá já passaram. Os assuntos tratados têm sido variados ci- figura 2 - Célula calorimétrica (em primeiro plano) com um dos computadores utilizados para a aquisição de dados. No écran pode verse uma curva típica de uma experiência calorimétrica.

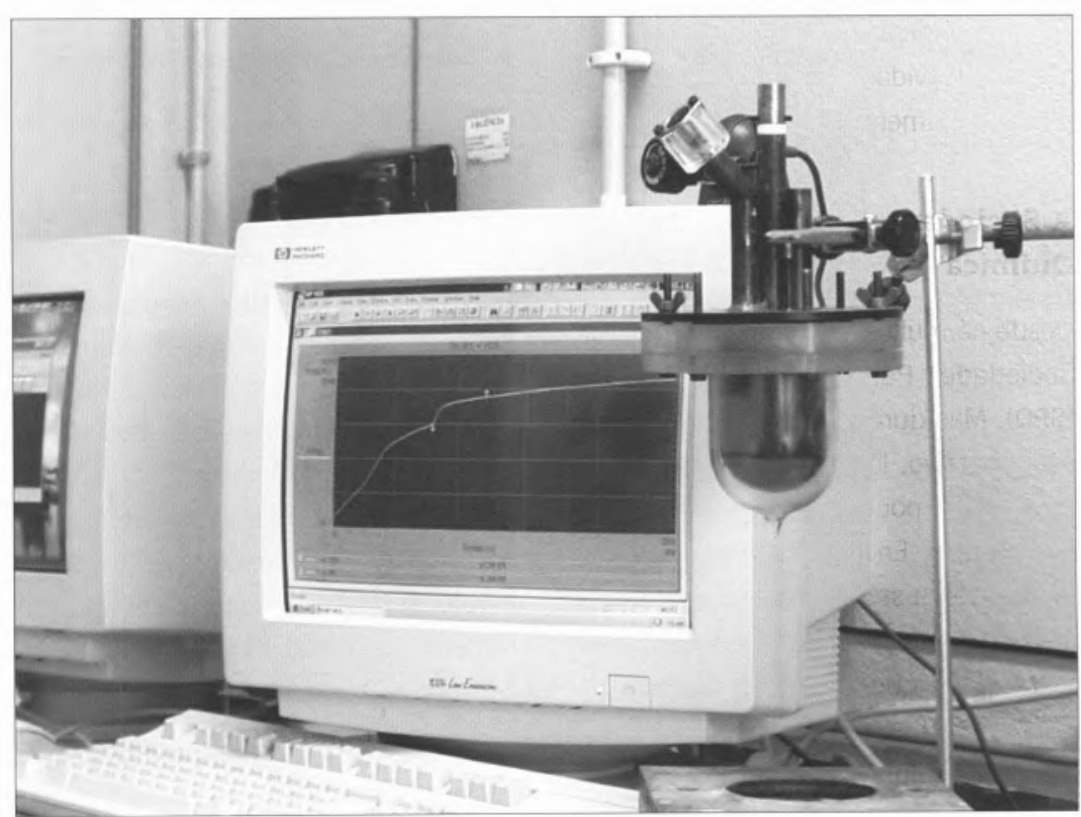




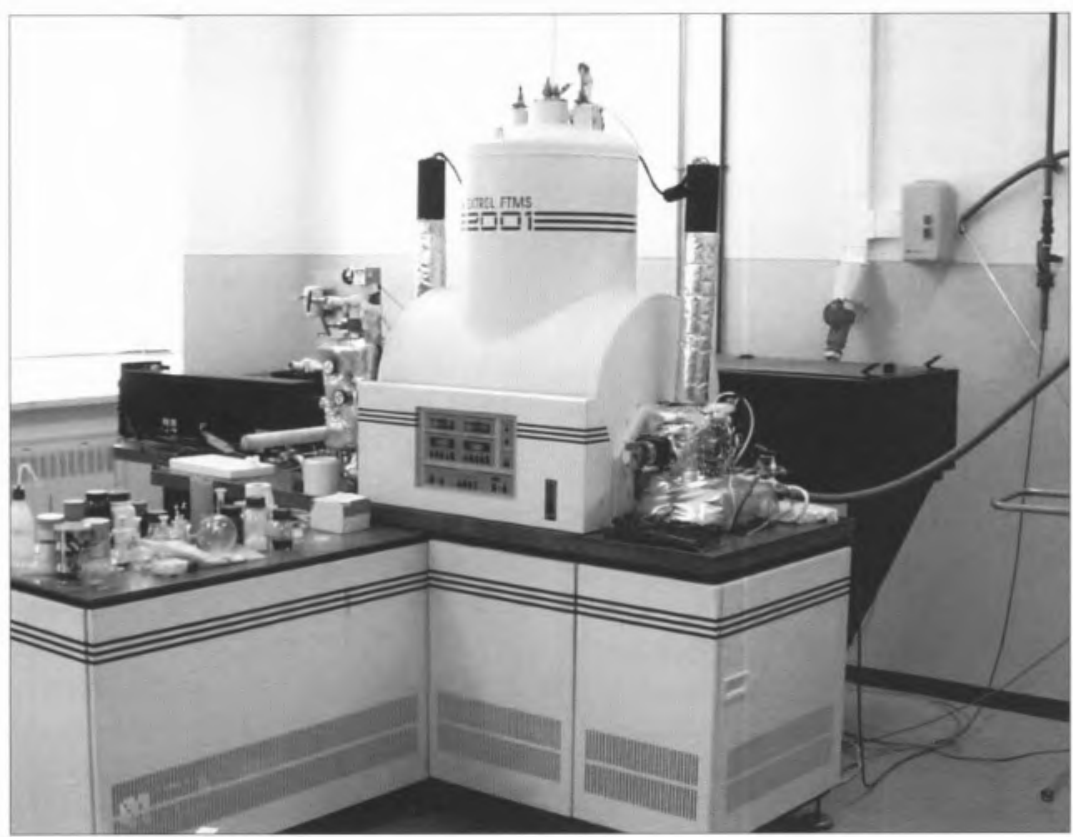

tando aqui apenas alguns para realçar a versatilidade da técnica em si e deste aparelho em particular: reactividade de iões de lantanídeos e actinídeos, e respectivos óxidos, com hidrocarbonetos [6] , acidez de antioxidantes do tipo fenol subtituído, desde os mais simples até à vitamina $\mathrm{E}$ [7], estudo de monoterpenos [8], análise de resíduos em anforas [9].

Neste momento continuam em estudo a acidez de fenóis polisubstituídos, a reactividade de iões de compostos de coordenação de metais de transição e, com merecido destaque pela sua inovação, os estudos que estão a ser actualmente levados a cabo pelo Doutor Joaquim Marçalo e pela Marta Santos sobre reactividade de iões de neptúnio, plutónio e amerício [10].

\section{A Sociedade Portuguesa de Química}

Desde há muitos anos que sou sócio da Sociedade Portuguesa de Química (SPQ). Mas durante muitos anos fui um sócio passivo, limitando-se a minha intervenção a pouco mais do que pagar a quota anual. Em 1991 o Prof. Martinho Simões torna-se Secretário Geral da Sociedade Portuguesa de Química e desafia-me para que eu monte na sede da $S P Q$ um clube de Software onde fosse possivel qualquer sócio consultar pro- gramas com interesse pedagógico e/ou educacional na área da Química antes de decidir da sua aquisição ou simplesmente para saber o que existia no mercado [11]. E deste modo, o meu gosto por computadores, foi canalizado para esta área. Pode parecer que hoje um tal projecto não faz sentido. Podemos testar as versões "demo" de um qualquer programa através da internet. Mas em 1991, não era assim. Para além do Clube de Software o meu interesse nessa área deu origem a uma série de artigos de critica de software educacional [12], a duas cadeiras, uma de mestrado e outra de licenciatura [13] que são lecionadas na FCUL e no começo de 2003 o Luis Rosa defenderá sob minha orientação a sua tese de mestrado intitulada "A Química na Net, a Net da Química" onde é feito um levantamento crítico de sites relacionados com o ensino da Química em língua portuguesa. O futuro nesta área ainda está por construir, mas terá que ser construído depressa para que não corramos o risco de ficar para trás.

\section{Comentário Final}

Não podia aqui esquecer uma das maiores realizações do Prof. Martinho Simões: conseguiu criar o "Grupo de Termoquímica de Lisboa", grupo esse com um modo de funcionamento único figura 3 - Vista geral do espectrómetro de massa de ressonância ião-ciclotrão. $\mathrm{O}$ cilindro horizontal contém, para além de azoto e hélio liquidos, o magneto supercondutor.

14] e que foi e continua a ser fundamental na minha actividade científica.

Acima de tudo o que foi dito, para além de todas as suas realizações pessoais (como sobejamente atesta o Perfil Biográfico publicado nesta revista), o que 0 Prof. Martinho Simões deixou e continua a deixar ainda hoje (e espero que por muitos anos) é uma imagem de marca, uma maneira própria e entusiasmada de fazer as coisas e de encarar os desafios e a ciência na sua globalidade, e a sua capacidade de transmitir a outros esse mesmo entusiasmo e vontade de enfrentar os desafios. Bem haja.

\section{Referências e notas}

[1] (a) "Uranium (III) Poly(pyrazol-1-yl)borate complexes", I. Santos, N. Marques, A. Pires de Matos, Inorganica Chimica Acta 1985 , 110, 149-151; (b) "Some reactions of Hydrotris(3,5-dimethylpyrazolyl)borato trichloroactinides(IV), $\mathrm{MCl}_{3}\left(\mathrm{HBL}_{3}\right)$.thf ( $\mathrm{M}=\mathrm{Th}^{\mathrm{IV}}, \mathrm{U}^{\mathrm{IV}}$; $\mathrm{L}=3,5$-dimethylpyrazolyl, thf=tetrahydrofuran)", J. Marçalo, N. Marques, A. Pires de Matos, K. W. Bagnall, J. Less-Common Metals 1986, 122, 219-224.

[2] "Uranium-oxygen and uranium-nitrogen bond dissociation enthalpies in U(IV) polipyrazolylborate complexes", J. P. Leal, N. Marques, A. Pires de Matos e J. A. Martinho Simões, "Italian-Portuguese-Spanish Meeting in Inorganic Chemistry (Towards Europe)", 
Gandia, Espanha, 1990, e "11th IUPAC Conference on Chemical Thermodynamics", Como, Itália, Agosto de 1990

[3] (a) "Standard enthalpies of formation of sodium alkoxides", J. P. Leal, A. Pires de Matos, J.A. Martinho Simōes, J. Organomet Chem. 1991, 403,1-10; (b) "Standard enthalpies of formation of lithium alkoxides", J. P. Leal, J. A. Martinho Simôes, J. Organomet. Chem. 1993, 460, 131-138; (c) "Standard molar enthalpies of formation of $\mathrm{Mg}$ and Ca alkoxides", T. Barreira, J. P. Leal, Eur. J. Inorg. Chem. 2000, 987-991; (d) "Standard Molar Enthalpies of Formation of Li, Na, K and TI Cyclopentadienyls", A. Carvalho, V. Cachata, J. P. Leal, Eur. J. Inorg. Chem. 2001, 1587-1591; 'Bonding Energetics in Alkaline Metal Alkoxides and Phenoxi des", P. Nunes, J. P. Leal, V. Cachata, H. Raminhos, and Manuel E. Minas da Piedade, submetido para publicação.

[4] (a) "Uranium-ligand bond dissociation enthalpies in U(IV) polipyrazolylborate complexes", J. P. Leal, N. Marques, A. Pires de Matos, M. J. Calhorda, A. M. Galvão e J.A. Martinho Simões, Organometallics 1992, 11, 1632-1637; (b) "Uranium-ligand bond dissociation enthalpies in U(IV) polypyrazolylborate complexes. II", J. P. Leal, J. A. Martinho Simões, J. Chem. Soc. Dalton Trans. 1994, 2687-2691; (c) "Bond Dissociation Enthalpies in U(IV) Complexes. An integrated view.", J. P. Leal, N. Marques, J. Takats, J. Organomet. Chem. 2001, 632, 209-214; (d) "Enthalpies of Formation of Europium Alkoxides", J. P. Leal, J. Branco, J. M. Carretas, "12 Tage der Seltenen Erden", Hamburgo, Alemanha, Dezembro de 1999.

[5] 0 programa de trabalhos intitula-se "Reactividade de Hidrocarbilos de U(IV) e Energética das Ligações Urânio-Carbono", tendo a Maria Augusta Antunes sido contemplada com uma bolsa de doutoramento por parte da FCT para a sua prosecussão.

[6] p. ex. (a) "Gas phase actinide ion chemistry: activation of alkanes and alkenes by thorium cations", J. Marçalo, J. P. Leal, A.
Pires de Matos, Int. J. of Mass Spectrom. and Ion Proc. 1996, 157/158, 265-274; (b) "Gas phase actinide ion chemistry: FTICR/MS study of the reactions of thorium and uranium metal and oxide ions with arenas", J. Marçalo, J. P. Leal, A. Pires de Matos, A. G. Marshall, Organometallics $1997,16,4581-4588$

[7] (a) "Experiências de equilíbrio ião-molécula num espectrómetro de massa de ressonância ião-ciclotrăo (FT-ICR)", J. P. Leal, M. T. N. Fernandez, J. A. Martinho Simōes, "2. Encontro Nacional de Química-Física", Porto Portugal, Junho de 1995; (b) "Acidez de fenóis na fase gasosa", H. Martins, J. P. Leal, T. Fernandez, "V Conferência de Química-Física", SPQ, Faro, Portugal, Setembro de 2001; (c) "Acidez de fenóis polissubstituídos na fase gasosa: a influência dos substituintes", H. Martins, M. T. Fernandez, J. P. Leal, "XVIII Encontro Nacional da Sociedade Portuguesa de Química" , Aveiro, Portugal, Março de 2002.

[8] "Chemical ionization mass spectrometry study of some monoterpenes", M. R. L. Carvalho, J. P. Leal, M. F. Duarte, M. T. Fernandez, "3. Encontro de Espectrometria de Massa da SPQ", Lisboa, Portugal, Setembro de 1997.

[9] "Laser desorption fourier transform mass spectrometric analysis of food residues from amphorae", J. M. P. Cabral, J. P. Leal e J. Marçalo, "Archaeometry 92 - 28th International Symposium on Archaeometry", Los Angeles, USA, 1992

[10] (a) "Gas-phase oxidation reactions of neptunium and plutonium ions investigated via Fourier Transform Ion Cyclotron Resonance Mass Spectrometry", M. Santos, J. Marçalo, A. Pires de Matos, J. K. Gibson, R. G. Haire, J. Phys. Chem. A 2002, 106, 7190-7194; (b) "FT-ICR/MS study of the gasphase thermochemistry of americium oxides“, M. Santos, J. Marçalo, J. P. Leal, A. Pires de Matos, J. K. Gibson, R. G. Haire, Int. J. Mass Spectrom., (submetido para publicação).
[11] (a) "O clube de Software da SPQ. I", J. P. Leal, Química 1994, 52, 60; (b) "O clube de Software da SPQ. II", J. P. Leal, Química $1997,64,54$

[12] (a)"Comentário do Programa 'Acid-Base Package, Chem. Ed. Software", J. P. Leal, M. J. Matos, Quimica 1993, 49, 42. (b) "Comentário do Programa 'Spec20, Chem. Ed. Software'", J. P. Leal, M. J. Matos, Quimica 1993, 50, 67; (c) "Comentário do Programa 'Alkimers, Chem. Ed. Software'", J. P. Leal, Química 1993, 51, 63; (d) "Comentário do Programa 'Le Chat, SoftCiência'", J. P. Leal, Química 1994, 54, 92; (e) "Comentário do Programa 'Sirius'", M. J. Matos, M. Portugal, J. P. Leal, Química 1995, 57, 65; (f) "Accord para Excel e Access (uma ferramenta química)", J. P. Leal, Química 1997, 66, 10; (g) "Análise do Programa 'CAChe for Windows", J. P. Leal, Química 1998, 70, 35; "Análise do Programa 'HSC Chemistry", J. P. Leal, Química 1999, 75, 16; (h) "Análise do Programa 'Le Chat 2', Maria Adelaide F. A. C. Ramos, Paula Amador Louçã, J. P. Leal, Química 2001, 80, 44.

[13] Cadeiras de "Recursos para o Ensino da Química" inserida no mestrado de Quimica para o Ensino, ministrado pelo Departamento de Química e Bioquímica da Faculdade de Ciências da Universidade de Lisboa, e de "Software Educacional" inserida como opção no $3 .^{\circ}$ ano da Licenciatura em Ensino da Física e da Química - Variante Química, da FCUL.

[14] 0 "Grupo de Termoquímica de Lisboa" é formado por três grupos, um no Instituto Superior Técnico, liderado pelo Doutor Manuel Eduardo Minas da Piedade, outro no Instituto Tecnológico e Nuclear, liderado por mim próprio, e um terceiro, na Faculdade de Ciências da Universidade de Lisboa, liderado pelos Doutor José Artur Martinho Simōes. Todos os assuntos científicos são discutidos mensalmente por todos os elementos, e a utilização das verbas atribuídas para a investigação é feita de um modo assaz flexivel. As próprias técnicas existentes nos vários locais são complementares.

Um ensino da ciência em que não se aprenda a pensar, não é um ensino da ciência, mas sim um ensino da subserviência. 